\title{
Characterization, antioxidant and cytotoxic activity of sulfated derivatives of a water-insoluble polysaccharides from Dictyophora indusiata
}

\author{
CHAO DENG ${ }^{1,2 *}$, JINGJING XU ${ }^{3 *}$, HAITIAN FU ${ }^{2}$, JINGHUA CHEN ${ }^{2}$ and $\mathrm{XIN} \mathrm{XU}^{4}$ \\ ${ }^{1}$ Wuxi Medical School and ${ }^{2}$ School of Pharmaceutical Science, Jiangnan University, Wuxi, Jiangsu 214122; \\ ${ }^{3}$ Clinical Laboratory, Wuxi No. 4 People's Hospital, Jiangnan University, Wuxi, Jiangsu 214000; \\ ${ }^{4}$ Cardiovascular Department, Wuxi No. 2 People's Hospital, Wuxi, Jiangsu 214002, P.R. China
}

Received January 27, 2014; Accepted November 7, 2014

DOI: $10.3892 / \mathrm{mmr} .2014 .3060$

\begin{abstract}
The present study described the characterization and biological properties of water-soluble sulfated polysaccharides prepared from water-insoluble polysaccharide (DIP), which were extracted from Dictyophora indusiata. The sulfation of DIP was performed using the chlorosulfonic acid-pyridine method. The water solubilities of the sulfated derivatives were measured at room temperature according to the Chinese Pharmacopoeia. The scavenging activity of hydroxyl radicals and 1,1-diphenyl-2-picrylhydrazyl (DPPH) as determined, together with the reduction ability of the sulfated polysaccharides. The cytotoxic and antiproliferative effects of DIP and the sulfated derivatives on MCF-7 and B16 cells were then determined using an MTT assay. The substitution degrees of the sulfated polysaccharides were 0.584 (S1-DIP), 0.989 (S2-DIP) and 1.549 (S3-DIP) according to barium chloride-gelatin nephelometry. Infrared spectroscopy and ${ }^{13} \mathrm{C}$-nuclear magnetic resonance indicated that the substitution of S-DIP occurred mainly at the C- 6 position, followed by the C- 4 and C-2 positions. A significant increase was noted in the antioxidant activity of the sulfated derivatives compared with that of DIP. In addition, the S-DIPs exhibited a more marked reducing capacity and clearing activity of hydroxyl radicals and DPPH. This indicated that the antioxidant capacity of the polysaccharides was significantly higher following sulfation. Furthermore, in in vitro cell investigations, DIP exhibited no
\end{abstract}

Correspondence to: Dr Jinghua Chen, School of Pharmaceutical Science, Jiangnan University, 1800 Lihu Road, Wuxi, Jiangsu 214122, P.R. China

E-mail: jscdcdj1@163.com

Dr Xin Xu, Cardiovascular Department, Wuxi No. 2 People's Hospital, 68 Zhongshan Road, Wuxi, Jiangsu 214002, P.R. China

E-mail: xinfirst1@163.com

*Contributed equally

Key words: Dictyophora indusiata, water-insoluble polysaccharide, sulfation, antioxidant activity, antitumor activity inhibitory effects on the growth of the B16 or MCF-7 tumor cells. However, the sulfated derivatives exerted marked inhibitory effects on these cell lines. Sulfate modification may therefore contribute to an improvement in water solubility and in the antioxidant and antitumor activities of natural DIP.

\section{Introduction}

Polysaccharides are biological macromolecules, which function as structural materials and energy sources in cell life cycles and are important in avoiding infection, in immune regulation and in antitumor, antioxidative and antiviral processes (1-5). Previous studies have focused on the activities of polysaccharides derived from the fruiting body of Dictyophora (D.) indusiata. In 1983, Ukai et al (6) reported that water-soluble (1-3)-beta-D-glucans isolated from $D$. indusiata had marked antitumor effects against subcutaneously implanted sarcoma 180 in mice. In addition, five homogeneous polysaccharides have been identified from the fruiting bodies of $D$. indusiata, together with a conjugated polysaccharide fraction, which exhibited significant mitogenic and colony stimulating factor-inducing activities (7). In a previous study by our group, PD3, a water-soluble polysaccharide (DIP) isolated from $D$. indusiata, was confirmed as a type of $\beta-(1-3)$-D-glucan with (1-6)- $\beta$-glucopyranoside side chains and was observed to inhibit S180 tumor growth in vivo. Subsequent investigation of an extract from $D$. indusiata collected in a different growing area revealed a polysaccharide with a similar structure to that of PD3 and exhibited antioxidant activity in vitro $(8,9)$. All these studies focussed on the physical and chemical attributes of water-soluble polysaccharides; however, few studies have been performed to investigate the chemical attributes of waterinsoluble polysaccharides from $D$. indusiata $(10,11)$.

It is well established that the physicochemical and biological properties of polysaccharides are closely associated with their structure (12) and pendent groups $(13,14)$. Therefore, molecular modification, including methylation, sulfation, hydroxyethylation, hydroxypropylation and phosphorylation contribute to the improvement of the physicochemical and biological properties of polysaccharides (15). Among these methods, sulfation has been considered as a straightforward approach for the modification of 
polysaccharide structure in order to improve the water-solubility and functional properties of polysaccharides. Bao et al (16) reported that sulfated alpha-D-glucan, an alkaline-extractable polysaccharide from Ganoderma iucidum with a higher degree of substitution, exhibited increased water solubility. In addition, Deng et al (17) demonstrated that sulfated polysaccharides from the fruiting body of Pleurotus tuber-regium induced inhibitory effects on the increase in rat mitochondria caused by ferrocyanide ion-vitamin $\mathrm{C}$ and provided protection against carbon tetrachloride-induced lipid peroxidation.

In the present study, DIP was obtained from D. indusiata. Subsequently, sulfated derivatives of this DIP were generated with different degrees of substitution. The present study aimed to investigate the effects of sulfation on water-solubility and on the in vitro antioxidant and antitumor activities of these derivatives to assist in their development as a potential therapeutic application.

\section{Materials and methods}

Materials and agents. The fruiting bodies of D. indusiata were purchased from Xinguan Ecological Agriculture Development Co., Ltd (Hubei, China). Dimethyl sulfoxide (DMSO), LiCl, EDTA, trypsin, MTT, 1,1-diphenyl-2-picrylhydrazyl (DPPH) and ascorbic acid were purchased from Sigma-Aldrich (St. Louis, MO, USA). RPMI-1640 medium and fetal bovine serum (FBS) were obtained from Invitrogen Life Technologies (Carlsbad, CA, USA). Standard monosaccharides, including glucose, xylose, rhamnose, arabinose, mannose and galactose were obtained from Sangon Biotech Co., Ltd. (Shanghai, China). Sepharose CL-6B was purchased from GE Healthcare (Little Chalfont, UK). MCF-7 human breast cancer cells and B16 mouse melanoma cells were obtained from the Cell Bank of the Chinese Academy of Sciences (Shanghai, China) and were authenticated by morphological characteristic checks under an inverted phase contrast microscope (Optec BDS200; Chongqing Optec Instrument Co., Ltd, Chongqing, China). Chlorosulfonic acid (CAS), pyridine (Pyr), $\mathrm{BaCO}_{3}$, hydroxylamine hydrochloride, acetic anhydride, ferrous sulfate $\left(\mathrm{FeSO}_{4}\right)$, hydrogen peroxide $\left(\mathrm{H}_{2} \mathrm{O}_{2}\right)$, salicylic acid, sodium phosphate, potassium ferricyanide, potassium bromide $(\mathrm{KBr})$ and other chemicals and solvents were of analytical grade and obtained from Sinopharm Chemical Reagent Co., Ltd (Shanghai, China).

Isolation and purification of DIP. DIP was isolated from the fruiting bodies of $D$. indusiata, as previously described (18). In brief, the dried fruiting bodies were crushed into powder and then defatted with ethyl acetate and ethanol for $6 \mathrm{~h}$ using a Soxhlet extraction apparatus (Sigma-Aldrich). Subsequently, the degreased powder was extracted using distilled water at $100^{\circ} \mathrm{C}$ until the water-soluble polysaccharide was completely removed. The residues were then dried and extracted using $5 \% \mathrm{NaOH}$ containing $0.05 \% \mathrm{NaBH}_{4}$ at $25^{\circ} \mathrm{C}$ for $2 \mathrm{~h}$ and the mixture was then centrifuged at $14,000 \mathrm{x}$ g for $30 \mathrm{~min}$. The supernatant was decanted and then neutralized with glacial acetic acid and the product was lyophilized on a vacuum freeze dryer (Free Zone 2.5 1, Labconco Co., Ltd, Kansas, MO, USA) to derive the DIPs. Sepharose CL-6B $(1 \mathrm{~cm} \times 100 \mathrm{~cm})$ was used to identify the homogenicity of the DIP. The column was eluted with $0.5 \mathrm{M} \mathrm{NaOH}$ at a flow rate of $0.5 \mathrm{ml} / \mathrm{min}$. Aliquots of the fractions $(3.0 \mathrm{ml})$ were then collected using a fraction collector. The total sugar content of all the fractions was detected using the phenol-sulfuric acid method (19).

Sulfation of DIP. Sulfation of DIP was performed using the chlorosulfonic acid (CAS)-pyridine (Pyr) method, as described previously (20). The degrees of substitution were controlled via a molar ratio of chlorosulfonic acid to monosaccharide residues, with ratios of 3:1 (S1-DIP), 4:1 (S2-DIP) and 5:1 (S3-DIP), respectively.

Water solubility assay. The water solubilities of the sulfated derivatives were measured at room temperature according to the Pharmacopoeia of the People's Republic of China (21).

Chemical analysis. The total sugar content was determined using the phenol-sulfuric acid method (19) with glucose as a standard, the uronic acid content was estimated using the sulfuric acid-carbazole method with glucuronic acid as a standard (22) and the sulfur content was determined using the barium chloride-gelatin method (23). The degree of substitution (DS) was calculated from the sulfur content (S) using the following formulas:

$$
\begin{gathered}
\mathrm{S}(\%)=\frac{\mathrm{SO}_{4}(\mu \mathrm{g}) \times 0.1374 \times 100}{\text { sample }(\mu \mathrm{g})} \\
\mathrm{DS}=\frac{162 \times \frac{\mathrm{S} \%}{32}}{100-\left(\frac{102}{32} \times \mathrm{S} \%\right)}
\end{gathered}
$$

Determination of monosaccharide compositions. The monosaccharide composition of DIP was determined using gas chromotography-mass spectrometry (GC-MS) (QP2010s; Shimadzu, Kyoto, Japan). Briefly, $20 \mathrm{mg}$ monosaccharide was hydrolyzed with $2 \mathrm{ml} 1 \mathrm{~mol} / \mathrm{l}$ sulfuric acid at $100^{\circ} \mathrm{C}$ for $4 \mathrm{~h}$. The residual sulfuric acid was removed by neutralization with excess $\mathrm{BaCO}_{3}$ following hydrolysis. The hydrolyzate was evaporated under reduced pressure, dissolved in $0.5 \mathrm{ml}$ pyridine and reacted with $10 \mathrm{mg}$ hydroxylamine hydrochloride at $90^{\circ} \mathrm{C}$ for $30 \mathrm{~min}$. Subsequently, $0.5 \mathrm{ml}$ acetic anhydride was added and incubated for $30 \mathrm{~min}$ at $90^{\circ} \mathrm{C}$. Following cooling down, the solution obtained was filtered with a $0.22-\mu \mathrm{m}$ organic filter and loaded onto a Rxi-1 ms capillary GC column (30 m x 0.25 mm x $0.25 \mu \mathrm{m}$; Restek Corp., Bellefonte, PA, USA) at $120-250^{\circ} \mathrm{C}$ at a rate of $5^{\circ} \mathrm{C} / \mathrm{min}$. Rhamnose, arabinose, xylose, mannose, glucose and galactose were used as monosaccharide standards.

Evaluation of molecular weight. The molecular weight (Mw) of the sample was determined by gel permeation chromatography (GPC) using the methods described in a previous study by our group (8). GPC measurements of the samples were performed on an Agilent 1200 LC (Agilent Technologies Inc., Santa Clara, CA, USA) with a pump G1310A equipped with PL aquagel OH column $(7.5 \mathrm{~mm}$ x $300 \mathrm{~mm})$ and differential refractive index detector $(\mathrm{G} 1362 \mathrm{~A})$ at $25^{\circ} \mathrm{C}$. The eluent was $\mathrm{NaNO}_{3}$ aqueous solution $(0.2 \mathrm{M})$, and the flow rate was set at $0.8 \mathrm{ml} / \mathrm{min}$. All the solutions were filtered with $0.45 \mu \mathrm{m}$ sand filter. 
Fourier transform infrared (FTIR) spectroscopy. FTIR spectroscopy was performed on a Nicolet Nesux 470 spectrophotometer (Thermo Scientific Corporation, Pittsburgh, PA, USA) at a wavenumber range between 4,000 and $400 \mathrm{~cm}^{-1}$ using the potassium bromide disc method (24). Briefly, samples mixed with potassium bromide were ground in an agate mortar for $10 \mathrm{~min}$, while the mass ratio of potassium bromide to added sample was $\sim 100-200$. Subsequently, the obtained powder was placed in casting equipment and compressed to form a disc, which was fixed to the sample holder and tested.

Nuclear magnetic resonance $(N M R)$ analysis. ${ }^{13} \mathrm{C}$ NMR analysis was performed at $400 \mathrm{MHz}$ using a Bruker Advance spectrometer (Aduance III 400MHz; Bruker Coporation, Madison, WI, USA). The DIP was dissolved in DMSO and the sulfated derivatives were dissolved in $\mathrm{D}_{2} \mathrm{O}$. The temperature was set at $70^{\circ} \mathrm{C}$.

Hydroxyl radical scavenging activity. Fenton's reaction was performed to determine the hydroxyl radical scavenging activity of the sulfated derivatives at concentrations of $25-1,000 \mu \mathrm{g} / \mathrm{ml}$ according to the previously described method (25). The reaction volume consisted of $0.2 \mathrm{ml}$ DIP and sulfated derivatives, $2.0 \mathrm{ml}$ EDTA-FeSO $(0.15 \mathrm{mM}), 2.0 \mathrm{ml} \mathrm{H} \mathrm{O}_{2}(6.0 \mathrm{mM}), 0.8 \mathrm{ml}$ salicylic acid $(2.0 \mathrm{mM})$ and $0.8 \mathrm{ml}$ distilled water. The hydroxyl radical was determined by monitoring the absorbance at $510 \mathrm{~nm}$ using a UV VIS spectrophotometer (UV-2550; Shimadzu, Kyoto, Japan) following incubation for $60 \mathrm{~min}$ at $37^{\circ} \mathrm{C}$. The hydroxyl radical scavenging activity was expressed according to the following formula:

$$
\text { Scavenging rate }(\%)=\frac{\mathrm{A}_{\text {sample510 }}-\mathrm{A}_{\text {control510 }}}{\mathrm{A}_{\text {blank510 }}-\mathrm{A}_{\text {control510 }}} \times 100
$$

where $\mathrm{A}_{\text {control510 }}$ was the absorbance obtained using water and sodium phosphate to replace the sample and $\mathrm{H}_{2} \mathrm{O}_{2}$, respectively; $\mathrm{A}_{\text {blank510 }}$ was the absorbance obtained using water to replace the sample and $\mathrm{H}_{2} \mathrm{O}_{2}$.

DPPH scavenging activity. The DPPH radical scavenging activity of the sulfated derivatives was measured using the previous description with minor modifications (26). Initially, the samples $(0.5 \mathrm{ml} ; 25-1,000 \mu \mathrm{g} / \mathrm{ml})$ were added to a $0.004 \%$ ethanol solution of DPPH $(2 \mathrm{ml})$. Following incubation at room temperature for $15 \mathrm{~min}$ in the dark, the absorbance at $517 \mathrm{~nm}$ was determined and the DPPH scavenging activity was calculated as follows:

$$
\text { Scavenging ability }(\%)=\frac{\mathrm{A}_{\text {blank517 }}-\mathrm{A}_{\text {sample517 }}}{\mathrm{A}_{\text {blank517 }}} \times 100
$$

where $A_{\text {blank517 }}$ was the absorbance obtained using water to replace the sample solution; and $\mathrm{A}_{\text {sample517 }}$ was the absorbance of polysaccharide solution.

Reduction ability. The reduction ability of the sulfated derivatives was determined according to the method reported by Yen and Chen (27) with slight modifications. Briefly, $1 \mathrm{ml}$ DIP and the sulfated derivatives (S1-DIP, S2-DIP and S3-DIP) in $2.5 \mathrm{ml}$ sodium phosphate buffer (2 M; pH 6.6) were mixed with $2.5 \mathrm{ml}$ aqueous potassium ferricyanide $(1 \% \mathrm{w} / \mathrm{v})$ and incubated at $50^{\circ} \mathrm{C}$ for $20 \mathrm{~min}$. Subsequently, $2.5 \mathrm{ml}$ trichloroacetic acid $(10 \%, \mathrm{w} / \mathrm{v})$ was added to terminate the reaction. The solution was then centrifuged at 3,000 xg for $10 \mathrm{~min}$, following which $2.5 \mathrm{ml}$ supernatant was mixed with $3.0 \mathrm{ml}$ distilled water and $0.2 \mathrm{ml}$ $0.1 \%$ ferric chloride $(\mathrm{w} / \mathrm{v})$. Absorbance at $700 \mathrm{~nm}$ was measured using a UV VIS spectrophotometer (UV-2550; Shimadzu).

Cytotoxic activity of sulfated polysaccharide. The MCF-7 cells were cultured in RPMI-1640 complete medium supplemented with 10\%FBS, $200 \mathrm{mmol} / 1$ glutamine, $100 \mathrm{U} / \mathrm{ml}$ penicillin, $100 \mu \mathrm{g} / \mathrm{ml}$ streptomycin and $5 \mu \mathrm{l} / \mathrm{ml}$ insulin. The B16 cells were cultured in RPMI-1640 complete medium supplemented with $10 \% \mathrm{FBS}, 200 \mathrm{mmol} / 1$ glutamine, $100 \mathrm{U} / \mathrm{ml}$ penicillin and $100 \mu \mathrm{g} / \mathrm{ml}$ streptomycin. All the cells were then incubated at $37^{\circ} \mathrm{C}$ in a humidified atmosphere with $5 \% \mathrm{CO}_{2}$.

To compare the antitumor effects of the DIP and the sulfated derivatives, $\mathrm{DMSO} / \mathrm{LiCl}$, a polar aprotic solvent that dissolves polar and nonpolar compounds, was added to dissolve the DIP. DMSO has been routinely used in biopreservation, particularly in cell cryopreservation; however, it is able to induce cell differentiation in the culturing process. Thus, the effects of different concentrations of $\mathrm{DMSO} / \mathrm{LiCl}$ on cell growth were investigated in the present study, which demonstrated that $\mathrm{DMSO} / \mathrm{LiCl}$ had no effects on cell growth at concentrations of $0.01-0.1 \%$ (data not shown). Therefore, the concentration of $\mathrm{DMSO} / \mathrm{LiCl}$ in the solvent used for DIPs in the in vitro experiments to determine tumor inhibitory effects was maintained at $<0.1 \%$.

MTT assay. The inhibitory effects of DIP on the cellular proliferation of MCF-7 and B16 cells were determined using an MTT assay. In brief, the MCF-7 or B16 cells were seeded

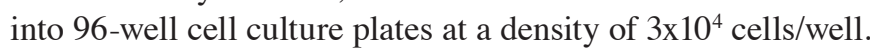
The DIP and its sulfated derivatives were dissolved in $0.05 \%$ (v/v) DMSO/LiCl and water, respectively. Following incubation for $24 \mathrm{~h}$, the cells were cultured with either DIP or the sulfated derivatives at concentrations of 25, 50, 100, 200, 500 and $750 \mu \mathrm{g} / \mathrm{ml}$. Cell lines treated with medium only were used as a control. Subsequently, $20 \mu \mathrm{l}$ MTT $(5 \mathrm{mg} / \mathrm{ml})$ was added to each well and plates were further incubated at $37^{\circ} \mathrm{C}$ for $4 \mathrm{~h}$. Following removal of the supernatant, $100 \mu \mathrm{l}$ DMSO was added to each well. The mixture was agitated in a horizontal direction for $10 \mathrm{~min}$ to dissolve the produced formazan crystals. The optical densities were measured at $570 \mathrm{~nm}$ using an ELISA microplate reader and each experiment was performed in triplicate. The cell viability was calculated as follows:

$$
\text { Inhibition rate }(\%)=\frac{\mathrm{Abs}_{\text {control }}-\mathrm{Abs}_{\text {sample }}}{\mathrm{Abs}_{\text {control }}} \times 100
$$

where $\mathrm{Abs}_{\text {sample }}$ was the absorbance of the cells treated with the samples, while $\mathrm{Abs}_{\text {control }}$ was the absorbance of the control cells.

Statistical analysis. Values are expressed as the mean \pm standard deviation. SPSS 13.0 software (SPSS, Inc., Chicago, IL, 
Table I. Physiochemical characteristics of sulfated polysaccharides derived from DIPs.

\begin{tabular}{lccc}
\hline $\begin{array}{l}\text { Product } \\
\text { code }\end{array}$ & DS & $\begin{array}{c}\text { Water solubility } \\
(\mathrm{mg} / \mathrm{ml})\end{array}$ & $\begin{array}{c}\text { Molecular weight, } \\
(\mathrm{KDa})\end{array}$ \\
\hline S1-DIP & 0.58 & 100.27 & 69 \\
S2-DIP & 0.99 & 109.89 & 74 \\
S3-DIP & 1.55 & 102.74 & 85 \\
\hline
\end{tabular}

DIP, water-insoluble polysaccharide; DS, degree of substitution.

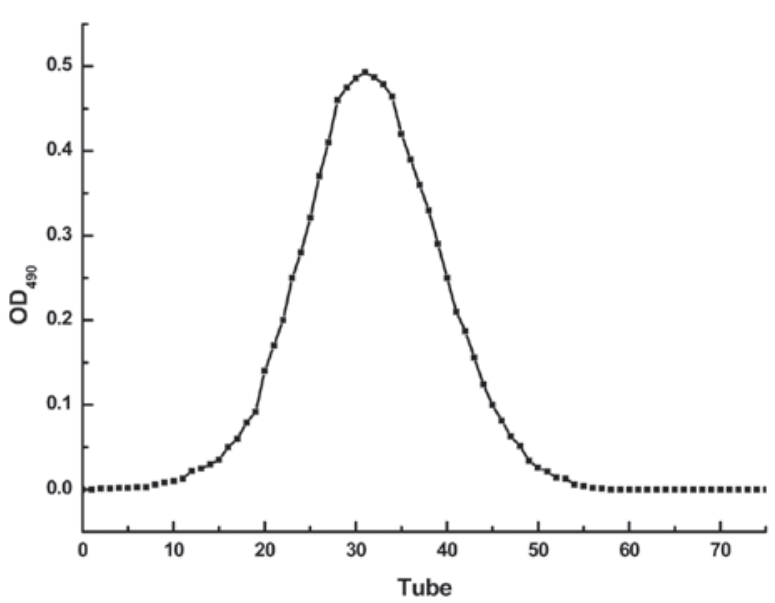

Figure 1. Sepharose CL-6B chromatogram of water-insoluble polysaccharides extracted from $D$. indusiata reveals a large peak close to aliquot 32 . The OD value at $490 \mathrm{~nm}$ indicated the content of carbohydrate using the phenol-sulfuric acid method assay. $\mathrm{OD}_{490}$, optical density at $490 \mathrm{~nm}$.

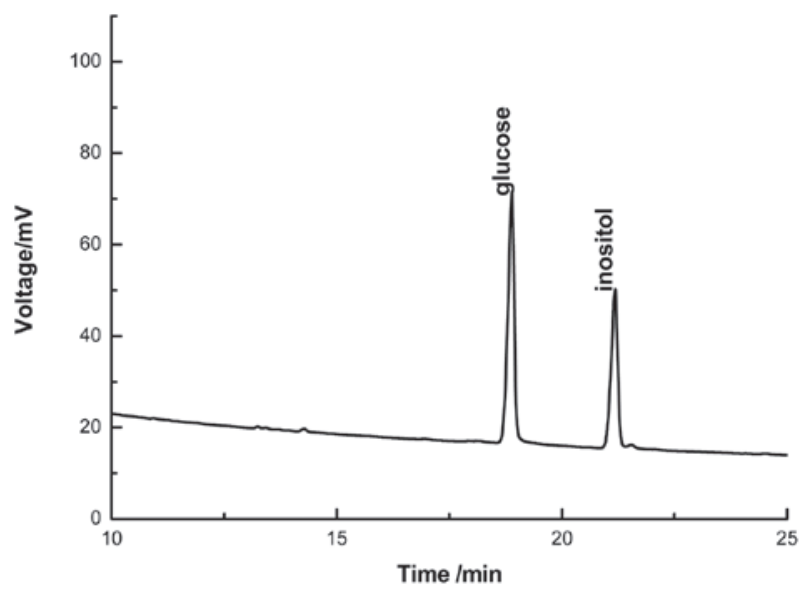

Figure 2. Gas chromatography spectrum of water-insoluble polysaccharides.

USA) was used for data analysis. One-way analysis of variance followed by the least significant difference test was performed for the inter-group comparison. $\mathrm{P}<0.05$ was considered to indicate a statistically significant difference.

\section{Results}

Properties and structural analysis of the DIP and sulfated derivatives. The final yield of DIP was $\sim 3.5 \%$ of the dried fruiting body powder. The tracer elution curve (Fig. 1) was single and symmetrical, which indicated purity of the DIP. The total sugar content and uronic acid content of DIP were 99.07 and $0.62 \%$, respectively (Table I), indicating that DIP was the neutral polysaccharide. In addition, the DIP was hydrolyzed to a monosaccharide by trifluoroacetic acid and further acetylated for GC-MS analysis. The results indicated that the DIP was a homopolysaccharide, which consisted only of glucose (Fig. 2).

The CAS-Pyr method was adapted to determine the synthesis of sulfated polysaccharide derivatives (S-DIP) in the present study (28). A total of three sulfated derivatives, including S1-DIP, S2-DIP and S3-DIP, with different DS were obtained from the DIP by controlling the molar ratio of chlorosulfonic acid to monosaccharide residues (Table II). The DS of S1-DIP, S2-DIP and S3-DIP ranged between 0.58 and 1.55. The Mw of S1-DIP, S2-DIP and S3-DIP was 69, 74 and $85 \mathrm{kDa}$, respectively. A marked increase was noted in the DS and $\mathrm{Mw}$ of the sulfated derivatives as the molar ratio of the chlorosulfonic acid to monosaccharide residues increased. Additionally, S1-DIP, S2-DIP and S3-DIP exhibited high levels of water solubility (Table II), indicating that sulfation improved the water solubility of the original polysaccharide.

The absorption of DIP ranged between 1,060 and $1,000 \mathrm{~cm}^{-1}$, which demonstrated that the monosaccharide of DIP was in a pyranose form. In addition, a characteristic absorption band at $842 \mathrm{~cm}^{-1}$ was observed, which was indicative of an $\alpha$-configuration of the glycosidic bond. Compared with DIP, two new absorption bands were observed in the spectra of sulfated derivatives at $1,240 \mathrm{~cm}^{-1}$ and $810 \mathrm{~cm}^{-1}$, which were characteristic of an asymmetric $\mathrm{S}=\mathrm{O}$ stretching vibration and a symmetric C-O-S vibration, respectively (29). Additionally, the absorption bands at $2,930 \mathrm{~cm}^{-1}$ and $1,350 \mathrm{~cm}^{-1}$, which stemmed from the $\mathrm{C}-\mathrm{H}$ stretching vibration, were weaker as the DS increased (Fig. 3). These changes in the IR peaks confirmed the sulfation of DIP.

The results of the ${ }^{13} \mathrm{C}-\mathrm{NMR}$ analysis were consistent with the IR substitution measurements. Due to its wide range of chemical shifts and limited overlapping peaks, ${ }^{13} \mathrm{C}-\mathrm{NMR}$ has been frequently used to examine polysaccharide structure. The NMR spectra of DIP and S-DIP are shown in Fig. 4. The substitution of a sugar ring on the polysaccharide caused changes in chemical shifts.

The ${ }^{13} \mathrm{C}-\mathrm{NMR}$ spectrum of DIP (Fig. 4A) contained six peaks at C-1 (100.2 ppm), C-2 (71.5 ppm), C-3 (83.1 ppm), C-4 (70.1 ppm), C-5 (72.6 ppm) and C-6 (60.8 ppm), which correlated with the carbons in $[\alpha-\text { Glc }(1 \rightarrow 3)-]_{n}$. In addition, the peak at $100.2 \mathrm{ppm}$ was due to due to an anomeric carbon chemical shift, which indicated that the polysaccharides were formed by glycosidic bonds. With the monosaccharide analysis and infrared spectrum in consideration, these results confirmed that DIP was an $\alpha$ - $(1 \rightarrow 3)$-D-glucan.

As shown in Fig. 4B and Table II, the $-\mathrm{OH}$ groups at the $\mathrm{C}-6$ position were substituted according to the peak areas of C-6 and C-6s, at 61.2 and 66.5 ppm, respectively. The peak at $61.2 \mathrm{ppm}$ was significantly weaker compared with that of the DIP signal and new signals appeared at 63.704-67.374 ppm, indicating that partial sulfation of C-6 had occurred. Additionally, as shown in Table II, substitution of S-DIP also occurred in the C-4 and C-2 positions. 
Table II. ${ }^{13} \mathrm{C}$ nuclear magnetic resonance chemical shifts of samples.

\begin{tabular}{|c|c|c|c|c|c|c|c|c|c|c|c|c|}
\hline Samples & $\begin{array}{c}\mathrm{C}-1 \\
(\mathrm{ppm})\end{array}$ & $\begin{array}{c}\text { C-1' } \\
(\mathrm{ppm})\end{array}$ & $\begin{array}{c}\mathrm{C}-2 \\
(\mathrm{ppm})\end{array}$ & $\begin{array}{l}\mathrm{C}-2 \mathrm{~s} \\
(\mathrm{ppm})\end{array}$ & $\begin{array}{c}\mathrm{C}-3 \\
(\mathrm{ppm})\end{array}$ & $\begin{array}{c}\mathrm{C}-4 \\
(\mathrm{ppm})\end{array}$ & $\begin{array}{c}\mathrm{C}-4^{\prime} \\
(\mathrm{ppm})\end{array}$ & $\begin{array}{c}\text { C-4s } \\
(\mathrm{ppm})\end{array}$ & $\begin{array}{c}\mathrm{C}-5 \\
(\mathrm{ppm})\end{array}$ & $\begin{array}{c}\mathrm{C}-6 \\
(\mathrm{ppm})\end{array}$ & $\begin{array}{l}\text { C-6s } \\
(\mathrm{ppm})\end{array}$ & Reference \\
\hline DIP & 100.2 & - & 71.5 & - & 83.1 & 70.1 & - & - & 72.6 & 60.8 & - & This study \\
\hline S-DIP & 99.9 & 98.2 & 71.5 & 77.4 & 85.5 & 70.5 & 70.2 & 73.2 & 73.5 & 61.2 & 66.5 & This study \\
\hline$[\alpha-\text { Glc }(1 \rightarrow 3)-]_{\mathrm{n}}$ & 100.6 & - & 71.1 & - & 83.2 & 70.6 & - & - & 72.8 & 61.1 & - & (18) \\
\hline
\end{tabular}

DIP, water-insoluble polysaccharide; S-, sulfated; $-2 \mathrm{~s},-4 \mathrm{~s},-6 \mathrm{~s}$, sulfated carbon atoms.

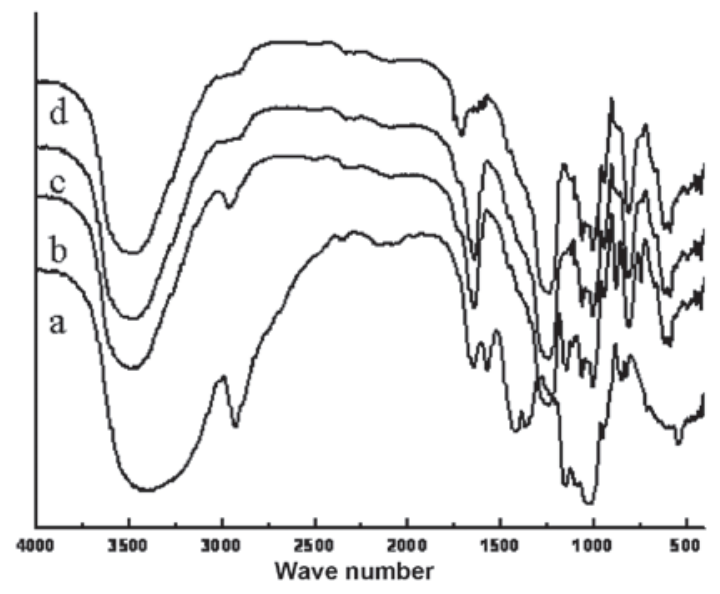

Figure 3. Infrared spectrum $\left(\mathrm{cm}^{-1}\right)$ of (a) DIP, (b) S1-DIP, (c) S2-DIP and (d) S3-DIP. DIP, water-insoluble polysaccharide; S-, sulfated.

Hydroxyl radical scavenging activity. As shown in Fig. 5A, the hydroxyl radical scavenging activity of S1-DIP, S2-DIP and S3-DIP was markedly higher compared with that of DIP at concentrations of $100,300,500$ and $1,000 \mu \mathrm{g} / \mathrm{ml}$, respectively. The scavenging activity of DIP was weak at $<15 \%$, including that at $1,000 \mu \mathrm{g} / \mathrm{ml}$. The hydroxyl radical scavenging activity of S1-DIP, S2-DIP and S3-DIP was concentration-dependent. Furthermore, S2-DIP with a DS value of 0.989 exhibited more marked scavenging of hydroxyl radicals compared with S1-DIP and S3-DIP, which had a DS of 0.584 and 1.549 , respectively. This suggested that the changes in molecular structure induced by sulfation may be associated with the scavenging activity of hydroxyl radicals in addition to DS. These results indicated that the sulfate group was important in the scavenging capacity of hydroxyl radicals.

DPPH scavenging activity. The DPPH assay was used as a substrate to determine the antioxidative activity of compounds. The DPPH scavenging activity of DIP and its derivatives is shown in Fig. 5B. It was noted that the scavenging capability of DPPH was enhanced as the concentrations of S1-DIP, S2-DIP and S3-DIP increased. However, no increase was observed in the scavenging activity of DPPH by DIP. Significant differences were observed in the DPPH scavenging activities of the S1-DIP, S2-DIP and S3-DIP groups compared with those of the DIP group at concentrations $>100 \mu \mathrm{g} / \mathrm{ml}(\mathrm{P}<0.05)$. The maximal scavenging activity was observed in S2-DIP (63.6\%), followed by S3-DIP (60.58\%)
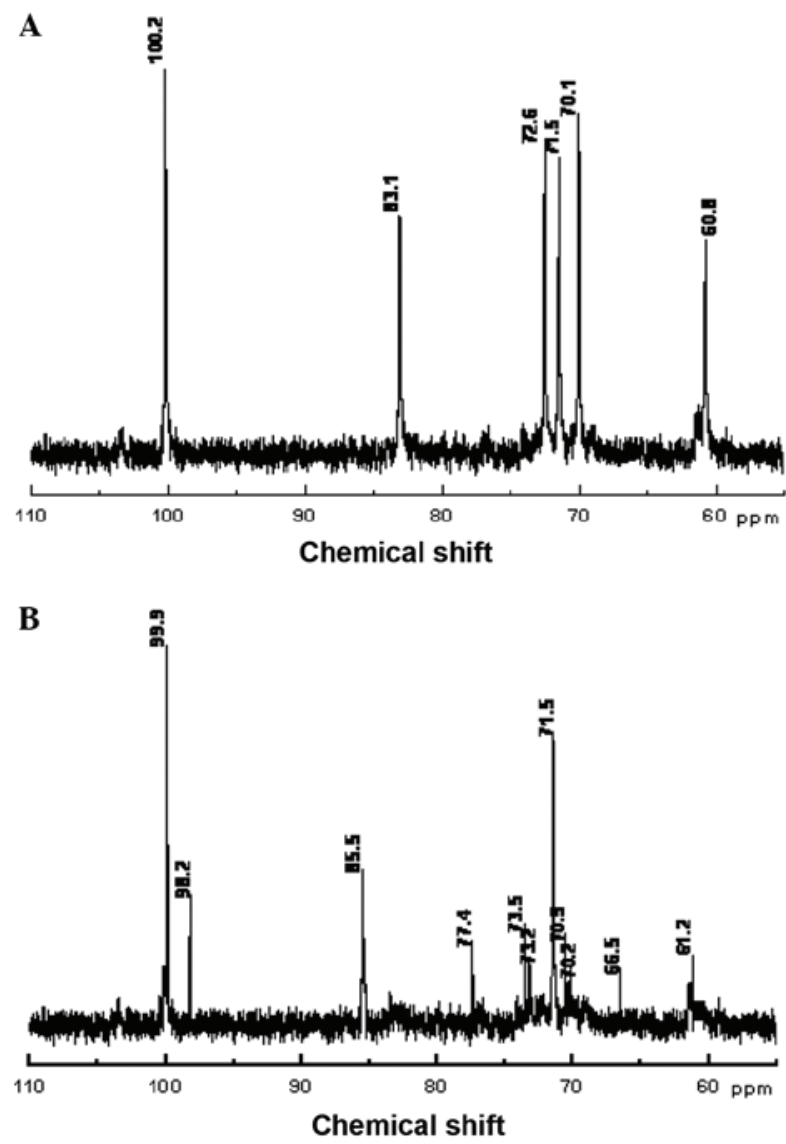

Figure $4 .{ }^{13} \mathrm{C}-\mathrm{NMR}$ spectrum of (A) DIP and (B) S-DIP. DIP, water-insoluble polysaccharide; S-, sulfated.

and S1-DIP (58.1\%) at 1,000 $\mu \mathrm{g} / \mathrm{ml}$. All sulfated derivatives exhibited a significantly higher scavenging effect compared with that of DIP $(\mathrm{P}<0.05)$. Overall, the results demonstrated that sulfation improved the antioxidant activity of DIP.

Reduction ability. The reduction ability of DIP and its sulfated derivatives is shown in Fig. 5C, which revealed that the absorbance value was positively associated with the reduction ability. The reduction ability increased as the concentrations of S1-DIP, S2-DIP and S3-DIP increased. However, no significant increase was observed in the reduction ability with increasing concentration of DIP. The reduction ability of S1-DIP, S2-DIP and S3-DIP was $0.645,0.705,0.675$ at a concentration of $1,000 \mu \mathrm{g} / \mathrm{ml}$, respectively, which was $\sim 6.0$-fold higher compared with that of DIP. In addition, 

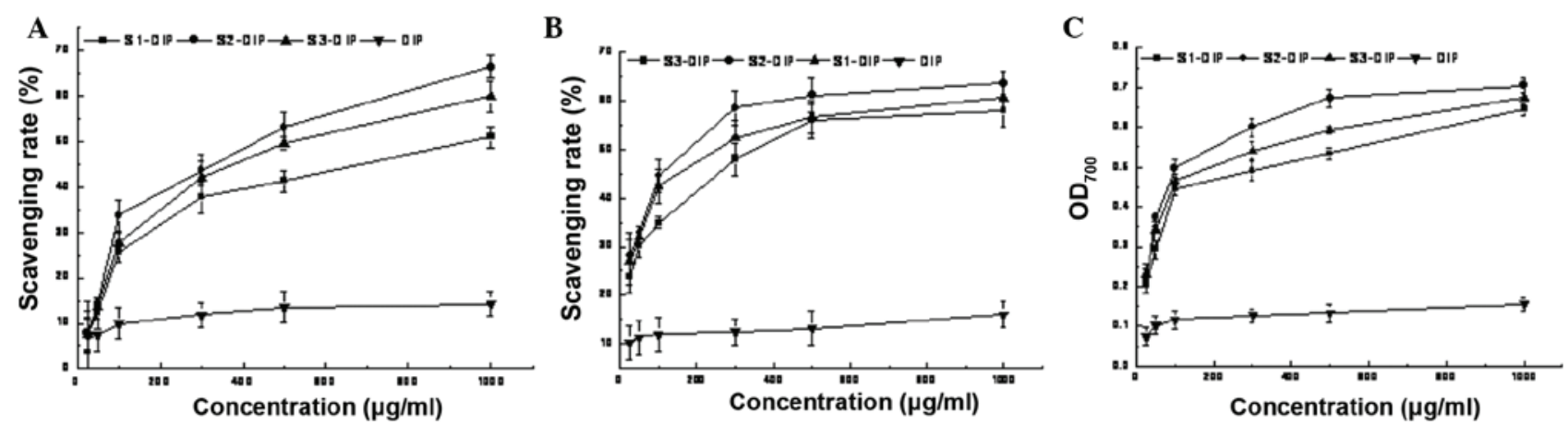

Figure 5. Comparison of the antioxidant activities of DIP and S-DIPs in (A) hydroxyl radical scavenging capacity, (B) 1,1-diphenyl-2-picrylhydrazyl radical scavenging capacity and (C) reduction ability. DIP, water-insoluble polysaccharide; S-, sulfated; $\mathrm{OD}_{700}$, optical density at $700 \mathrm{~nm}$.
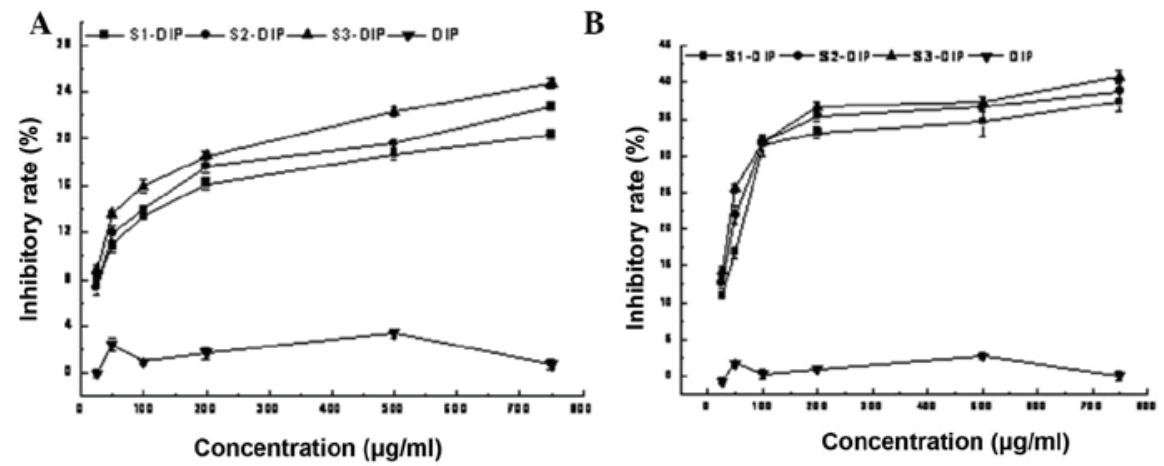

Figure 6. S-DIP-mediated inhibition of the growth of (A) MCF-7 cells and (B) B16 cells. DIP, water-insoluble polysaccharide; S-, sulfated.

compared with the absorbance rate of DIP, higher absorbance values were obtained for S1-DIP, S2-DIP and S3-DIP at the same concentration. Furthermore, significant differences were noted in the reduction potential among the different DS of the sulfated polysaccharides at $300-500 \mu \mathrm{g} / \mathrm{ml}(\mathrm{P}<0.05)$. The maximum reduction ability was observed for S2-DIP. These results indicated that sulfation enhanced the reduction potential of the original polysaccharide.

Inhibitory effects of sulfated polysaccharides. The inhibitory effects of DIP and S-DIPs on MCF-7 cells are shown in Fig. 6A. The S-DIPs exhibited greater inhibitory effects compared with that of DIP $(\mathrm{P}<0.05)$, while the unmodified DIP had no effect on the growth of the MCF-7 cells. At concentrations of 200-750 $\mu \mathrm{g} / \mathrm{ml}$, statistically significant differences were noted for the inhibitory effects on the MCF-7 cells between S1-DIP, S2-DIP and S3-DIP $(\mathrm{P}<0.05)$. Additionally, these inhibitory effects were enhanced with increasing DS.

In the B16 cells (Fig. 6B), no significant difference was identified in cell growth following treatment with DIP at concentrations of $25-750 \mu \mathrm{g} / \mathrm{ml}$. However, a significant inhibition was noted in the cell growth of the cells treated with the sulfated derivatives $(\mathrm{P}<0.05)$. Additionally, the inhibitory effects were more marked as the DS and concentration increased. S3-DIP exerted its maximum inhibitory effect at a concentration of $750 \mu \mathrm{g} / \mathrm{ml}$.

\section{Discussion}

In the present study, DIP was isolated from the fruiting bodies of D. indusiata and the physiochemical properties of the sulfated derivatives, including water-solubility, and in vitro antioxidant and antitumor activities, were investigated. The results of the present study may provide useful information for the investigaton of natural medicines with therapeutic applications.

Using the CAS-Pyr method, the synthesis of the S-DIP was performed in the present study and the results were consistent with those of a previous study, in which the authors described the degradation of polysaccharide in acidic environments (30). Previous studies have suggested that sulfation is associated with increased water solubility of polysaccharide derived from Grifola frondosa (31) and the derivatives of $20(S)$-ginsenoside Rh2 (32). In the present study, enhanced water solubility was also observed in the sulfated derivatives compared with the DIP. It was hypothesized that this may be associated with the structural changes induced by sulfation. However, further studies are required to confirm this hypothesis.

Of note, the sulfation of DIP may be associated with an increase in antitumor activity via the addition of sulfate groups to the polysaccharide (33). It has been proposed that the sulfated group may result in a relatively expanded and stiff polysaccharide chain in aqueous solutions. Additionally, 
it has been demonstrated that a high level of chain stiffness and a satisfactory water solubility of sulfated derivatives is beneficial for increased antitumor effects $(34,35)$. In the present study, the antitumor effects of sulfated derivatives on MCF-17 and B16 cells were investigated, which revealed significant inhibition of cell growth following treatment with sulfated derivatives compared with those treated with DIP $(\mathrm{P}<0.05)$. In addition, the inhibitory effects were more marked with increasing DS and concentration. All these results demonstrated that the sulfated polysaccharide, derived from the fruiting bodies of $D$. indusiata, exhibited significant antitumor activities. The present study hypothesized that the mechanism may be associated with direct cytotoxicity to the MCF-17 and B16 cells. In addition, other studies have demonstrated that the sulfation of polysaccharides may be associated with modulation of the host immune system, which contributes to the improvement of the host immunity suppressed by the tumor cells $(36,37)$. The aim of further investigation is to focus on the cytotoxicity of sulfated derivatives on the antitumor effects in vivo.

Several studies have been performed to determine the free radical scavenging activities of polysaccharide extracted from fungi. In 1997, Liu et al (38) reported that certain mushroom polysaccharide extracts had superoxide and hydroxyl radical scavenging activities. Luo and Fan (39) reported that polysaccharide extracted from Polygonum multiflorum exhibited powerful scavenging activities, particularly on DPPH and hydroxyl radicals. In the present study, the scavenging activities of hydroxyl radicals and DPPH were also examined. The results revealed that the scavenging activities of S1-DIP, S2-DIP and S3-DIP on hydroxyl radicals were concentration-dependent and superior to that of DIP. The maximum DPPH scavenging activity was observed for S2-DIP, followed by S3-DIP and S1-DIP. With regard to the reduction ability, significant differences were noted among the different sulfated polysaccharides. Compared with S1-DIP and S3-DIP, S2-DIP was more effective in the reduction ability, hydroxyl radical and DPPH scavenging activity assays. This was consistent with previous studies suggesting that a moderate DS of sulfated derivatives may be necessary for a high level of antioxidant activity $(20,40,41)$.

A water-insoluble polysaccharide was obtained from the body of $D$. indusiata using an alkaline extraction. The structural characterization indicated that DIP was a pure $\alpha$-D-glucan. A total of three sulfated derivatives, including S1-DIP, S2-DIP and S3-DIP with different DS were prepared using the chlorosulfonic acid-pyridine method. Compared with DIP, the sulfated derivatives S1-DIP, S2-DIP and S3-DIP exhibited higher antioxidant and antitumor activities. In addition, the sulfated derivatives were more effective in scavenging of hydroxyl radicals and DPPH, and had an increased reduction ability compared with DIP. No direct correlation was observed between the DS of the sulfated derivatives and antioxidant activity. In addition, sulfated derivatives had a significant inhibitory effect on growth of B16 or MCF-7 cells.

In conclusion, the present study demonstrated that sulfate modification may be an effective approach to improve the water solubility of DIP and can also enhance antioxidant and antitumor activities. Further studies are required to confirm the biological activities of the sulfated derivatives of DIP to provide a basis on which to investigate their potential application in medicinal or functional food.

\section{Acknowledgements}

This study was financially supported by the Doctoral Scientific Fund Project of the Ministry of Education of China (no. 20110093110008).

\section{References}

1. Eo SK, Kim YS, Lee CK and Han SS: Possible mode of antiviral activity of acidic protein bound polysaccharide isolated from Ganoderma lucidum on herpes simplex viruses. J Ethnopharmacol 72: 475-481, 2000.

2. Wang J, Xu X, Zheng H, et al: Structural characterization, chain conformation, and morphology of a beta-(1 $\rightarrow 3)$-D-glucan isolated from the fruiting body of Dictyophora indusiata. J Agric Food Chem 57: 5918-5924, 2009.

3. Albert O, Toubas D, Strady C, et al: Reactivity of $(1 \rightarrow 3)$-beta-d-glucan assay in bacterial bloodstream infections. Eur J Clin Microbiol Infect Dis 30: 1453-1460, 2011.

4. Liu F, Ooi VE, Liu WK and Chang ST: Immunomodulation and antitumor activity of polysaccharide-protein complex from the culture filtrates of a local edible mushroom, Tricholoma lobayense. Gen Pharmacol 27: 621-624, 1996.

5. Hetland G: Anti-infective action of immuno-modulating polysaccharides (beta-glucan and Plantago Major L. Pectin) against intracellular (Mycobacteria sp.) and extracellular (Streptococcus Pneumoniae sp.) respiratory pathogens. Curr Med Chem-Anti-Infective Agents 2: 135-146, 2003.

6. Ukai S, Kiho T, Hara C, et al: Polysaccharides in fungi. XIII. Antitumor activity of various polysaccharides isolated from Dictyophora indusiata, Ganoderma japonicum, Cordyceps cicadae, Auricularia auricula-judae, and Auricularia species. Chem Pharm Bull (Tokyo) 31: 741-744, 1983.

7. Hara C, Kumazawa Y, Inagaki K, et al: Mitogenic and colony-stimulating factor-inducing activities of polysaccharide fractions from the fruit bodies of Dictyophora indusiata Fisch. Chem Pharm Bull (Tokyo) 39: 1615-1616, 1991.

8. Deng C, Fu H, Teng L, et al: Anti-tumor activity of the regenerated triple-helical polysaccharide from Dictyophora indusiata. Int J Biol Macromol 61: 453-458, 2013.

9. Deng $\mathrm{C}, \mathrm{Hu} \mathrm{Z}, \mathrm{Fu} \mathrm{H}$, et al: Chemical analysis and antioxidant activity in vitro of a $\beta$-D-glucan isolated from Dictyophora indusiata. Int J Biol Macromol 51: 70-75, 2012.

10. Martens DA: Plant residue biochemistry regulates soil carbon cycling and carbon sequestration. Soil Biol Biochem 32:361-369, 2000.

11. Jiao G, Yu G, Zhang J and Ewart S: Chemical structures and bioactivities of sulfated polysaccharides from marine algae. Mar Drugs 9: 196-223, 2011.

12. Saitô H, Yoshioka Y, Uehara N, et al: Relationship between conformation and biological response for $(1 \rightarrow 3)$-beta-D-glucans in the activation of coagulation factor $\mathrm{G}$ from limulus amebocyte lysate and host-mediated antitumor activity. Demonstration of single-helix conformation as a stimulant. Carbohydr Res 217: 181-190, 1991.

13. Qi H, Zhao T, Zhang Q, et al: Antioxidant activity of different molecular weight sulfated polysaccharides from Ulva pertusa Kjellm (Chlorophyta). J Appl Psychol 17: 527-534, 2005.

14. Zhang Z, Zhang Q, Wang J, et al: Preparation of the different derivatives of the low-molecular-weight porphyran from Porphyra haitanensis and their antioxidant activities in vitro. Int J Biol Macromol 45: 22-26, 2009.

15. Ríos JL: Chemical constituents and pharmacological properties of Poria cocos. Planta Med 77: 681-691, 2011.

16. Bao X, Duan J, Fang X and Fang J: Chemical modifications of the $(1 \rightarrow 3)$-alpha-D-glucan from spores of Ganoderma lucidum and investigation of their physicochemical properties and immunological activity. Carbohydr Res 336: 127-140, 2001.

17. Deng CH, Yang XL, Gu XM, et al: A beta-D-glucan from the sclerotia of Pleurotus tuber-regium (Fr.) Sing. Carbohydr Res 328: 629-633, 2000.

18. Zhang P, Zhang L and Cheng S: Chemical Structure and Molecular Weights of $\alpha-(1 \rightarrow 3)$-D-Glucan from Lentinus edodes. Biosci Biotechnol Biochem 63: 1197-1202, 1999. 
19. DuBois M, Gilles K, Hamilton J, Rebers P and Smith F: Colorimetric method for determination of sugars and related substances. Anal Chem 28: 350-356, 1956.

20. Wang J, Guo H, Zhanga J, et al: Sulfated modification, characterization and structure-antioxidant relationships of Artemisia sphaerocephala polysaccharides. Carbohydrate Polymers 81: 897-905, 2010.

21. The Pharmacopoeia Commission of PRC. Pharmacopoeia of People's Republic of China. vol I, Chemical Industry Press, Beijing, pp17-18, 2005.

22. Bitter T and Muir HM: A modified uronic acid carbazole reaction. Anal Biochem 4: 330-334, 1962.

23. Tabatabai M and Bremner J: Arylsulfatase activity of soils Soil Sci Soc Am Proc 34: 225-229, 1970.

24. Sachdeva A and Cai S: Structural differences of proteins between solution state and solid state probed by attenuated total reflection Fourier transform infrared spectroscopy. Appl Spectrosc 63 458-464, 2009.

25. Yang XM, Yu W, Ou ZP, et al: Antioxidant and immunity activity of water extract and crude polysaccharide from Ficus carica L. fruit. Plant Foods Hum Nutr 64: 167-173, 2009.

26. Braca A, De Tommasi N, Di Bari L, et al: Antioxidant principles from Bauhinia tarapotensis. J Nat Prod 64: 892-895, 2001.

27. Yen $\mathrm{G}$ and Chen $\mathrm{H}$ : Antioxidant activity of various tea extracts in relation to their antimutagenicity. J Agric Food Chem 43: 27-32, 1995.

28. Lu Y, Wang D, Hu Y, Huang X and Wang J: Sulfated modification of epimedium polysaccharide and effects of the modifiers on cellular infectivity of IBDV. Carbohydr Polym 71: 180-186, 2008

29. Liu Y, Liu C, Tan H, et al: Sulfation of a polysaccharide obtained from Phellinus ribis and potential biological activities of the sulfated derivatives. Carbohydrate Polymers 77: 370-375, 2009.

30. Wang J, Zhang J, Wang X, et al: A comparison study on microwave-assisted extraction of Artemisia sphaerocephala polysaccharides with conventional method: Molecule structure and antioxidant activities evaluation. Int J Biol Macromol 45: 483-492, 2009.
31. Nie X, Shi B, Ding Y and Tao W: Preparation of a chemically sulfated polysaccharide derived from Grifola frondosa and its potential biological activities. Int $\mathrm{J}$ Biol Macromol 39: 228-233, 2006.

32. Fu BD, Bi WY, He CL, et al: Sulfated derivatives of 20 (S)-ginsenoside Rh2 and their inhibitory effects on LPS-induced inflammatory cytokines and mediators. Fitoterapia 84: 303-307, 2013.

33. Du Y, Gu G, Hua Y, et al: Synthesis and antitumor activities of glucan derivatives. Tetrahedron 60: 6345-6351, 2004.

34. Arafuka S, Koshiba N, Takahashi D and Toshima K: Systematic synthesis of sulfated oligofucosides and their effect on breast cancer MCF-7 cells. Chem Commun (Camb) 50: 9831-9834, 2014.

35. Murata J, Saiki I, Nishimura Si, Nishi N, Tokura S and Azuma I: Inhibitory effect of chitin heparinoids on the lung metastasis of B16-BL6 melanoma. Jpn J Cancer Res 80: 866-872, 1989.

36. Wasser SP: Medicinal mushrooms as a source of antitumor and immunomodulating polysaccharides. Appl Microbiol Biotechnol 60: 258-274, 2002.

37. Zheng R, Jie S, Hanchuan D and Moucheng W: Characterization and immunomodulating activities of polysaccharide from Lentinus edodes. Int Immunopharmacol 5: 811-820, 2005.

38. Liu F, Ooi VE and Chang ST: Free radical scavenging activities of mushroom polysaccharide extracts. Life Sci 60: 763-771, 1997.

39. Luo A and Fan Y: In vitro Antioxidant of a Water-Soluble Polysaccharide from Dendrobium fimhriatum Hook.var. oculatum Hook. Int J Mol Sci 12: 4068-4079, 2011.

40. Yan JK, Wang WQ, Ma HL and Wu JY: Sulfation and enhanced antioxidant capacity of an exopolysaccharide produced by the medicinal fungus Cordyceps sinensis. Molecules 18: $167-177,2012$.

41. Wang X, Wang J, Zhang J, Zhao B, Yao J and Wang $Y$ Structure-antioxidant relationships of sulfated galactomannan from guar gum. Int J Biol Macromol 46: 59-66, 2010. 